\title{
WEAKLY CONFLUENT MAPPINGS AND FINITELY-GENERATED COHOMOLOGY
}

\author{
JAMES T. ROGERS, JR. ${ }^{1}$
}

\begin{abstract}
In this paper we answer a question of Wayne Lewis by proving that if $X$ is a one-dimensional, hereditarily indecomposable continuum and if $H^{1}(X)$ is finitely generated, then $C(X)$, the hyperspace of subcontinua of $X$, has dimension 2.
\end{abstract}

Let $C(X)$ be the hyperspace of subcontinua of the continuum $X$ with the topology determined by the Hausdorff metric. A classical theorem of J. L. Kelley [4] asserts that if $X$ is a hereditarily indecomposable continuum and the dimension of $X, \operatorname{dim} X$, exceeds one, then $\operatorname{dim} C(X)=\infty$. On the other hand, E. D. Tymchatyn [9] has proved that if $X$ is a nondegenerate hereditarily indecomposable subcontinuum of the plane (hence $\operatorname{dim} X=1$ ), then $C(X)$ can be embedded in $R^{3}$, and consequently $\operatorname{dim} C(X)=2$.

Carl Eberhart and Sam Nadler, Jr. [1], and later Howard Cook, asked if there exists a one-dimensional, hereditarily indecomposable continuum with an infinitedimensional hyperspace. Wayne Lewis [7] has recently given such an example. Lewis' powerful technique yields a continuum with infinitely-generated cohomology, and he has asked (cf. [6]) if a one-dimensional continuum with finitely-generated (co)homology can have an infinite-dimensional hyperspace. In this note, we prove the following theorem.

THEOREM 1. If $X$ is a one-dimensional, hereditarily indecomposablecontinuum and $H^{1}(X)$ is finitely generated, then $\operatorname{dim} C(X)=2$.

Theorem 1 is equivalent, by an observation [1] of Eberhart and Nadler, to the following theorem.

THEOREM 2. If $X$ is a one-dimensional, hereditarily indecomposable continuum, if $H^{1}(X)$ is finitely generated, and if $f: X \rightarrow Y$ is a monotone, open mapping onto a nondegenerate continuum $Y$, then $\operatorname{dim} Y=1$.

Theorem 2 will follow as a corollary to the following theorem.

Received by the editors April 3, 1979; presented to the Society, January 3, 1980.

AMS (MOS) subject classifications (1970). Primary 54F45, 54F50; Secondary 54C10.

Key words and phrases. Weakly confluent, monotone, dimension, hyperspace, hereditarily indecomposable continuum.

${ }^{1}$ The author was partially supported by a research grant from the Tulane University Senate Committee on Research. 0002-9939/80/0000-0129/\$01.75 
THEOREM 3. If $X$ is a one-dimensional continuum with finitely-generated cohomology, and if $f: X \rightarrow Y$ is a weakly confluent mapping onto a nondegenerate continuum $Y$, then $Y$ is one-dimensional.

Recall that a mapping $f: X \rightarrow Y$ between continua is weakly confluent if for each subcontinuum $W$ of $Y$, there exists a subcontinuum $V$ of $Y$ such that $f(V)=W$. A. Lelek introduced the definition of weakly confluent maps in [5], where the reader may find additional information concerning such maps. The class of weakly confluent maps includes all monotone maps, all open maps and all confluent maps.

In this paper, cohomology is Čech cohomology with integral coefficients.

The simple proof of Theorem 3 begins along lines surveyed previously by Howard Cook. Namely, recall the following theorem of Mazurkiewicz [8], which may be phrased as follows.

THEOREM 4. If $X$ is a continuum and $\operatorname{dim} X>1$, then there exists a weakly confluent mapping of $X$ onto the unit square, $I \times I$.

Let $S$ denote the plane continuum consisting of all points in the plane having polar coordinates $(r, \theta)$ for which $r=1, r=2$, or $r=\left(2+e^{\theta}\right) /\left(1+e^{\theta}\right)$. M. K. Fort, Jr. [2] has proved the following theorem about $S$.

THEOREM 5. The pseudo-arc cannot be mapped onto $S$.

One notes that Fort's proof holds true for any continuum $X$ such that $\left[X, S^{1}\right]=$ 0 ; here $\left[X, S^{1}\right]$ denotes the homotopy classes of maps of $X$ into $S^{1}$.

Now suppose $H^{1}(X)$ is finitely generated with $n$ generators. Consider $n+1$ disjoint homeomorphic copies of $S$ contained in $I \times I$. Call them $S_{1}, \ldots, S_{n+1}$. According to Theorem 4, if $\operatorname{dim} Y>1$, there exists a weakly confluent mapping $g$ : $Y \rightarrow I \times I$ of $Y$ onto $I \times I$. Let $Y_{1}, \ldots, Y_{n+1}$ be subcontinua of $Y$ such that $g\left(Y_{i}\right)=S_{i}$. Let $X_{1}, \ldots, X_{n+1}$ be subcontinua of $X$ such that $f\left(X_{i}\right)=Y_{i}$.

It follows from the generalized Theorem 5 that $\left[X_{i}, S^{1}\right] \neq 0, i=1, \ldots, n+1$. Note that by Bruschlinsky's theorem, $\left[X_{i}, S^{1}\right]=H^{1}\left(X_{i}\right)$.

Consider the following part of the long exact sequence of the pair $\left(X, \cup_{i=1}^{n+1} X_{i}\right)$ :

$$
H^{1}(X) \stackrel{j^{*}}{\rightarrow} H^{1}\left(\bigcup X_{i}\right) \stackrel{\delta}{\rightarrow} H^{2}\left(X, \bigcup X_{i}\right)
$$

Since $X$ is one-dimensional, it follows from [3, p. 152] that $H^{2}\left(X, \cup X_{i}\right)=0$. Hence $H^{1}\left(\cup X_{i}\right)=\operatorname{ker} \delta=\operatorname{im} j^{*}$ by exactness. This equality is impossible, however, because $H^{1}(X)$ has only $n$ generators while

$$
H^{1}\left(\bigcup_{i=1}^{n+1} X_{i}\right) \cong \prod_{i=1}^{n+1} H^{1}\left(X_{i}\right)
$$

Therefore, $\operatorname{dim} Y=1$, and the theorem is proved.

Note that the Menger universal curve, a one-dimensional continuum, admits monotone maps onto continua of arbitrary high dimension, even infinite dimension [10]. The cohomology of the universal curve is, of course, not finitely generated. 


\section{REFERENCES}

1. C. Eberhart and S. B. Nadler, Jr., The dimension of certain hyperspaces, Bull. Acad. Polon. Sci. Sér. Sci. Math. Astronom. Phys. 19 (1971), 1027-1034.

2. M. K. Fort, Jr., Images of plane continua, Amer. J. Math. 81 (1959), 541-546.

3. W. Hurewicz and J. Wallman, Dimension theory, Princeton Univ. Press, Princeton, N. J., 1948.

4. J. L. Kelley, Hyperspaces of a continuum, Trans. Amer. Math. Soc. 52 (1942), 22-36.

5. A. Lelek, A classification of mappings pertinent to curve theory, Proc. Univ. of Oklahoma Topology Conf., University of Oklahoma, Norman, 1972, pp. 97-103.

6. Wayne Lewis, Dimensons of hereditarily indecomposable contimua, Proc. Geometric Topology Conf., Warsaw, 1978 (to appear).

7. __ Monotone maps of hereditarily indecomposable continua, Proc. Amer. Math. Soc. 75 (1979), 361-364.

8. S. Mazurkiewicz, Sur l'existence des continus indécomposables, Fund. Math. 25 (1935), 327-328.

9. E. D. Tymchatyn, Hyperspaces of hereditarily indecomposable plane continua, Proc. Amer. Math. Soc. 56 (1976), 300-302.

10. David Wilson, Open mappings of the universal curve onto continuous curves, Trans. Amer. Math. Soc. 168 (1972), 497-515.

Department of Mathematics, Tulane University, New Orleans, Louisiana 70118 